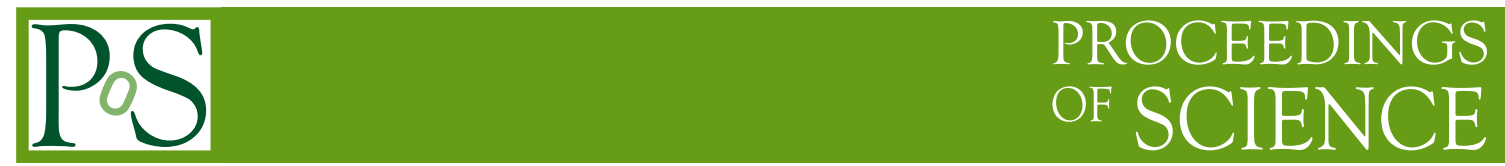

\title{
First measurements with the ALICE detector at LHC
}

\section{Domenico ELIA*广}

INFN Sezione di Bari, Bari (Italy)

E-mail: Domenico.Elia@ba.infn.it

\begin{abstract}
ALICE is a general-purpose detector designed to measure the properties of strongly interacting matter created in heavy-ion collisions at the CERN LHC. Several features, such as low momentum cut-off and powerful tracking over a broad momentum range, make it also an important contributor to the proton-proton LHC physics: here ALICE aims both at setting the baseline for the understanding of the heavy-ion data and exploring the new energy domain. This paper describes the status and plans for first physics measurements, in particular discussing the early p-p and $\mathrm{Pb}-\mathrm{Pb}$ running scenarios and the corresponding physics programmes.
\end{abstract}

The 2009 Europhysics Conference on High Energy Physics,

July 16 - 222009

Krakow, Poland

\footnotetext{
${ }^{*}$ Speaker.

${ }^{\dagger}$ On behalf of the ALICE Collaboration.
} 


\section{ALICE experiment at LHC}

The Large Hadron Collider (LHC) at CERN is expected to provide first p-p collisions by the end of 2009. The first run, starting with very few data at injection energy $(\sqrt{s}=0.9 \mathrm{TeV})$, will be mainly based on proton-proton collisions at $7 \mathrm{TeV}$ centre-of-mass energy and will last till the end of 2010 when a first pilot $\mathrm{Pb}-\mathrm{Pb}$ run should take place (10\% of the effective time corresponding to about $10^{6}$ seconds). ALICE (A Large Ion Collider Experiment) is the LHC experiment specifically devoted to the physics of ultra-relativistic heavy-ion collisions and the study of the quark-gluon plasma (QGP) phase[1, 2]. However, some features of the experimental apparatus in terms of design and performance will allow it to uniquely contribute to the p-p LHC physics as well[3].

The ALICE experimental apparatus has been designed as a dedicated heavy-ion detector optimized to measure a large variety of observables in very high multiplicity environments (up to 4000 charged particles per unit of rapidity with performance checked up to 8000). It will be able to detect and identify hadrons, leptons and photons over a wide range of momenta. The whole detector, shown in Figure 1, consists of a central part $(|\eta|<0.9)$ to detect hadrons, electrons and photons, a forward spectrometer to measure muons and additional smaller forward detectors for event characterization and triggering. A detailed description of the apparatus can be found in $[2,4]$.
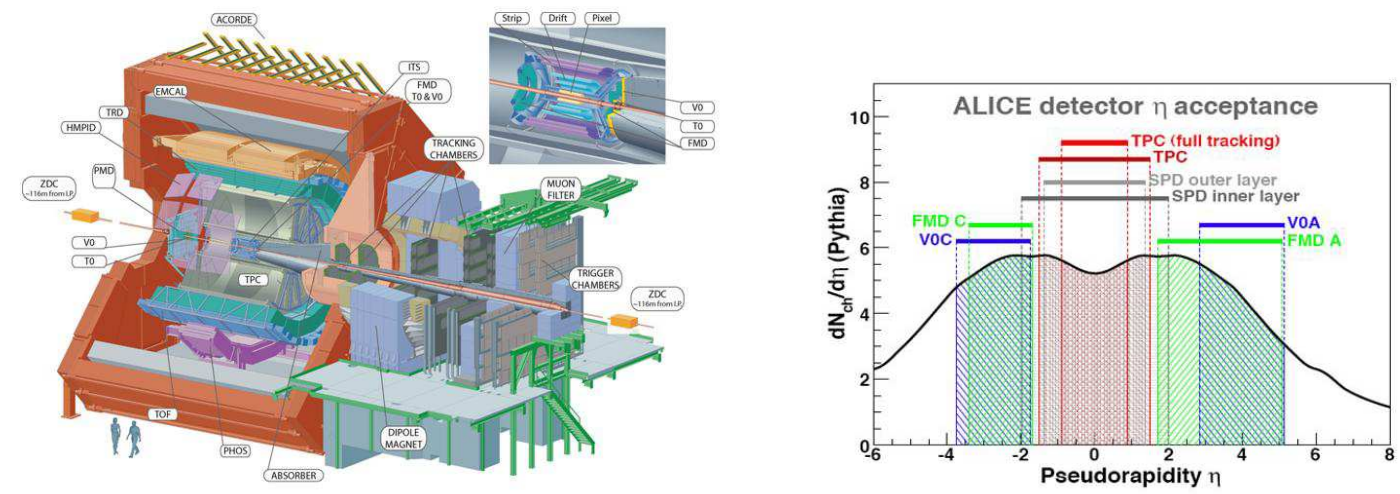

Figure 1: Left panel: General view of the ALICE detector and detail view (top right) of the ITS. Right panel: Acceptance coverage in $\eta$ for SPD, TPC and some of the forward detectors.

The Silicon Pixel Detector (SPD), the Time Projection Chamber (TPC) and the V0 detector will play a key role for the first data: as most of the other sub-systems, they are fully installed and commissioned[5]. The SPD is the innermost element of the ALICE Inner Tracking System (ITS), consisting of two layers of hybrid silicon pixels surrounding the beam pipe at 3.9 and 7.6 $\mathrm{cm}$ average radii with a total of $\approx 10^{7}$ pixel cells. It features a very low material budget $(\approx 1 \%$ per layer), a detection efficiency above $99 \%$, a spatial resolution of $\approx 12 \mu \mathrm{m}$ in the bending plane and a prompt signal as input to the level 0 trigger[4]. The SPD will allow the measurement of the charged particle multiplicity and pseudo-rapidity density distributions with a low momentum cut-off $(\approx 35$ $\mathrm{MeV} / c$ at $0.5 \mathrm{~T}$ field) up to $|\eta| \approx 2$. Furthermore, the so-called "Fast-OR" digital pulses coming from each of the 1200 SPD chips (indicating at least one pixel hit within the chip) will contribute to the minimum bias trigger and allow triggering on high multiplicity events[3,6].

The TPC has a cylindrical sensitive volume with radii between 85 and $250 \mathrm{~cm}$ for a length of $500 \mathrm{~cm}$ : it is the largest in the world and has been optimized for high track densities. It can track 
particles in $|\eta|<0.9$ with efficiency above $90 \%$, excellent momentum resolution up to $100 \mathrm{GeV} / c$ and particle identification up to $1 \mathrm{GeV} / c$. The $\mathrm{V} 0$ detector consists of a pair of tiled scintilator disks on either side of the interaction point: it will provide the minimum bias trigger (combined with the SPD Fast-OR) together with beam-gas background rejection and luminosity information[3].

\section{First proton-proton physics}

As already mentioned above, ALICE interest for the p-p LHC programme goes beyond the need to provide reference data for $\mathrm{Pb}-\mathrm{Pb}$. Its unique detection capabilities (tracking to a very low $\mathrm{p}_{T}$, excellent particle identification, very low mass tracking system) will allow to address a number of important studies within the p-p physics. The first proton-proton data taking scenario (approximately 10 months, starting by the end of 2009) is mostly based on collisions at $\sqrt{s}=7 \mathrm{TeV}$ with a luminosity of about $10^{30} \mathrm{~cm}^{-2} \mathrm{~s}^{-1}$ : in such conditions a statistics from few times $10^{8}$ to $10^{9}$ minimum bias events is expected to be collected. At the start-up some collisions at $0.9 \mathrm{TeV}$ should also be delivered (with a reduced luminosity of few times $10^{27} \mathrm{~cm}^{-2} \mathrm{~s}^{-1}$ ): this would be very useful to compare with existing measurements and estimate systematic uncertainties. The efficient minimum bias event trigger will allow ALICE to perform inclusive studies aimed at QCD measurements as those mentioned in the following.

With a statistics of few $10^{4}$ events (first few days, with a multiplicity reach up to $\approx 5$ times the mean multiplicity) a measurement of the charged particle pseudo-rapidity density and multiplicity distributions can be already performed. This can be achieved using the information of the SPD only and the corresponding tracklet reconstruction method[7]: with respect to the corresponding measurement based on the fully reconstructed tracks (ITS+TPC) it requires much faster alignment and calibration procedures being then suitable to extract results from the very first data. In the left panel of Figure 2 the acceptance correction for the SPD tracklets is shown. In the right panel the charged pseudo-rapidity distribution reconstructed with the SPD tracklets is compared, at the different correction stages, with the input distribution in p-p collisions generated with PYTHIA.
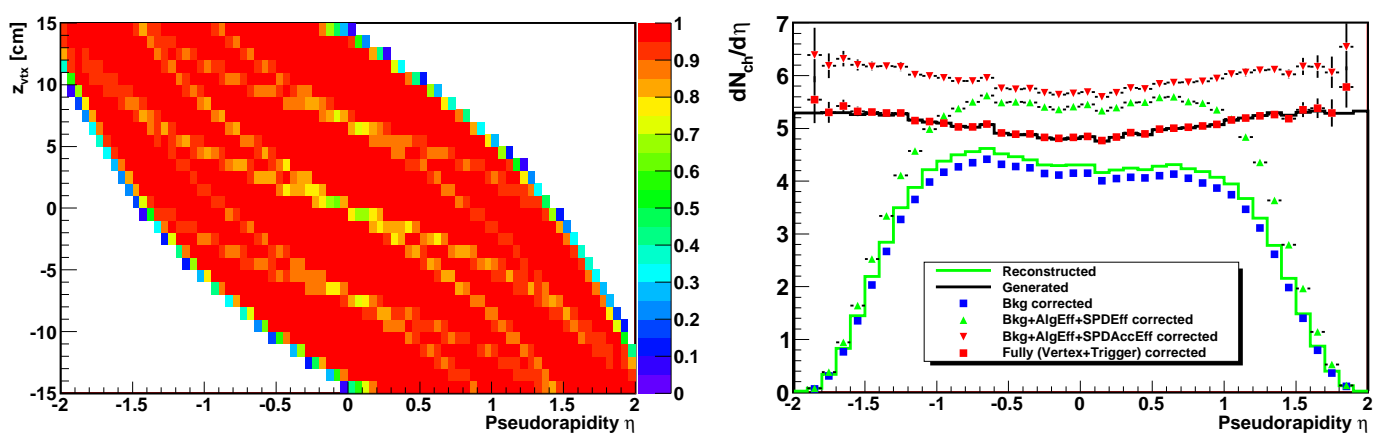

Figure 2: Left panel: Acceptance correction for the SPD tracklets as a function of $\eta$ and main vertex longitudinal position. Right panel: Reconstructed charged pseudo-rapidity density distributions at all the main correction stages, compared with the generated PYTHIA distribution in p-p at $7 \mathrm{TeV}$.

As charged particle multiplicity and pseudo-rapidity density correspond to basic properties of the collisions in the new energy domain at LHC, their knowledge will allow to tune the Monte Carlo generators. Moreover, the measurement of the charged-particle pseudo-rapidity density in 
the central rapidity region will extend the existing energy dependence pattern. Besides these very first measurements, $\mathrm{p}_{T}$ spectra of both all charged and identified particles, baryon number transport and strangeness production $\left(\mathrm{K}^{ \pm}, \mathrm{K}_{s}^{0}, \Lambda\right.$ and $\left.\bar{\Lambda}\right)$ analyses will also be carried out within the p-p first physics programme.

\section{Early heavy-ion physics}

The first heavy-ion run is scheduled for $5.5 \mathrm{~A} \mathrm{TeV} \mathrm{Pb}-\mathrm{Pb}$ collisions at reduced luminosity $\mathscr{L} \simeq 5 \times 10^{25} \mathrm{~cm}^{-2} \mathrm{~s}^{-1}$, corresponding to $1 / 20$ of the design luminosity. Running for $10^{6} \mathrm{~s}$ should be enough to collect $10^{7}$ minimum bias and another $10^{7}$ central $(5 \%)$ collisions. For this data taking a fully commissioned detector is expected: in particular alignment and calibrations will be available from the previously collected cosmics and p-p samples. Data quality and statistics should already allow with this pilot run to explore a quite rich physics spectrum. The initial $10^{5}$ events will provide information about global event properties such us multiplicity, pseudo-rapidity density and elliptic flow. Indeed the very first measurement in the ALICE heavy-ion physics programme will be the charged particle multiplicity density at mid-rapidity, followed by its behaviour along the $\eta$ range covered by the apparatus. Left panel of Figure 3 shows a collection of multiplicity results from heavy-ion and $\mathrm{p}-\overline{\mathrm{p}}$ collisions, where the A-A data are rescaled by the number of pairs of nucleons participating in the collision[2].
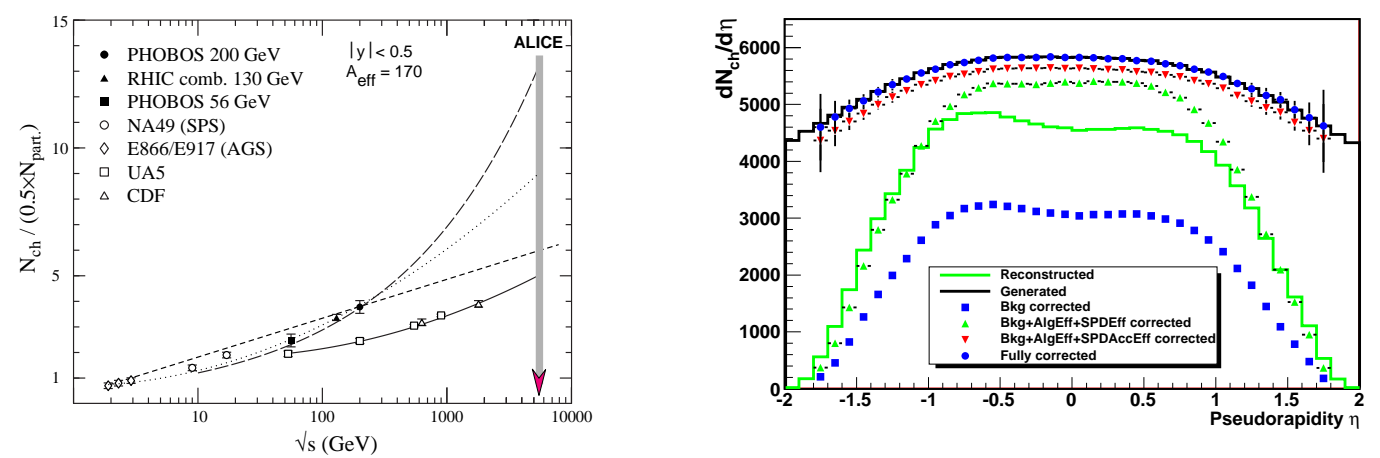

Figure 3: Left panel: Charged particle rapidity density per participant pair as a function of centre-of-mass energy for A-A and p-p collisions. Long dashed line is an extrapolation to LHC energies based on the saturation model. Right panel: Reconstructed charged particle pseudo-rapidity density distributions at all the main correction stages, compared with the generated HIJING distribution in central $\mathrm{Pb}-\mathrm{Pb}$ collisions at $5.5 \mathrm{~A} \mathrm{TeV}$.

When extrapolating from existing data to LHC energies there is a striking difference between the results obtained applying a saturation model[8] (long dashed line) or a fit in $\ln ^{2} \sqrt{s}$ (dotted line). The expected values for the $\mathrm{d} N_{c h} / \mathrm{d} \eta$ plateau level range from 1200 to 2600 (for most central 5\% collisions), substantially lower than the ALICE design value. In the right panel of Figure 3 an example of the charged particle pseudo-rapidity density reconstruction via SPD tracklets for $\mathrm{Pb}-\mathrm{Pb}$ collisions generated with Hijing is shown.

With a factor 10 more statistics $\left(10^{6}\right.$ events) particle spectra, resonances, differential flow and interferometry analyses will be reasonably accessible. The copious multiplicity of produced particles will allow to address essential measurements such as the particle composition and the 
transverse momentum distributions of identified particles. As an example, ALICE will have reconstruction rates of $13,0.1$ and 0.01 per event for the $\Lambda, \Xi$ and $\Omega$ hyperons respectively. The excellent performance in terms of tracking, vertexing and particle identification capabilities will be key factors: in Figure 4 displaced vertices from a cascade decay and an axample of $\pi \mathrm{p}$ invariant mass distribution with the $\Lambda$ peak in simulated central $\mathrm{Pb}-\mathrm{Pb}$ collisions are shown.
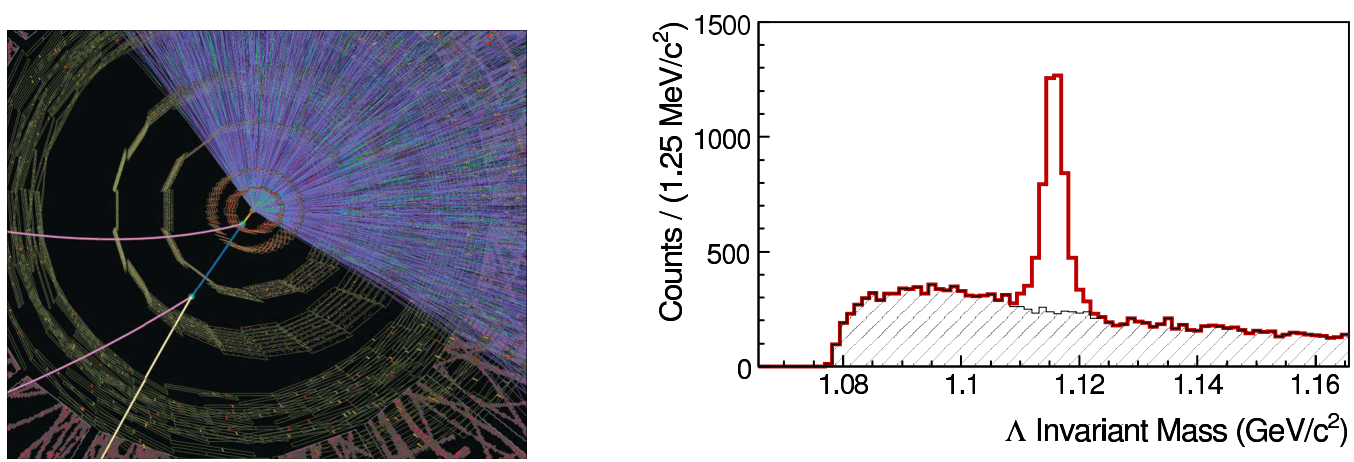

Figure 4: Left panel: fraction of $\mathrm{Pb}-\mathrm{Pb}$ event in the ITS with displaced vertices from a cascade decay. Right panel: $\pi$ p invariant mass distribution with the $\Lambda$ peak in central $\mathrm{Pb}-\mathrm{Pb}$ collisions.

Statistically significant samples of only $10^{6}$ events will provide freeze-out temperature and collective motion of the particle emitting source and allow to verify the thermal models which have successfully described hadron production up to RHIC energies[9]. In addition, bulk properties of the medium (jet quenching), heavy-flavours and charmonia production will also be achieved with a full sample of $10^{7}$ events from such first $\mathrm{Pb}-\mathrm{Pb}$ pilot run[3].

\section{Summary and outlook}

The ALICE experiment at LHC will collect first $\mathrm{p}-\mathrm{p}$ and $\mathrm{Pb}-\mathrm{Pb}$ data from the end of the year 2009 along the whole 2010. Measurements aiming to the global characterization of the $p-p$ collisions will be already accessible within the first few days of data taking. The expected detector performance and running scenarios should allow both in the first proton run and in the following pilot heavy-ion run a quite rich and uniquely interesting physics programme to be covered.

\section{References}

[1] ALICE Technical Proposal, CERN/LHCC 95-71 (1995).

[2] F. Carminati et al., ALICE: Physics Performance Report Volume I, J. Phys G 30 (2004) 1517.

[3] B. Alessandro et al., ALICE: Physics Performance Report Volume II, J. Phys G 32 (2006) 1295.

[4] K. Aamodt et al., JINST 3 (2008) S08002.

[5] P. Giubellino et al., these Proceedings.

[6] J. Conrad et al., ALICE-INT-2005-025 (2005).

[7] D. Elia et al., ALICE-INT-2009-021 (2009).

[8] K. J. Eskola et al., Nucl. Phys. B570 (2000), 379.

[9] A. Andronic et al., Nucl. Phys. A772 (2006), 167. 